\title{
Motion au Congrès international de Botanique à Bru- xelles 1910, relative à la nomenclature des Algues.
}

Par le Dr. S. Stockmayer (Unterwaltersdorf, N.-Ö.).

J'ai l'honneur à proposer:

Le point de départ pour la nomenclature des Algues (les Flagellatae et Characeae exclues) sera l'oeuvre:

J. B. De Toni, Sylloge Algarum omnium hucusque cognitarum, Patavii,

c'est à dire pour les:

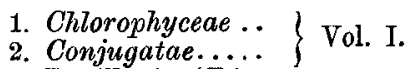

publié le

3. Bacillariae (Dia-

tomaceae $). . . . . . \quad$ Vol. II.

4. Phaeophyceae... Vol. III.

5. Rhodophyceae.. Vol. IV.

6. Myxophyceae

(Schizo-, Cyano-

phyceae)........ Vol. $\nabla . \quad$ digessit A. Forti

25. Juillet 1889

25. Juillet 1891

Sectio I. (Rhaphideae) Sectio II. (Pseudorhaphideae

Sectio III. Cryptorhaphideae

12. Février 1892

28. Avril 1894

14. Novembre 1895

14. Novembre 1897

2. Janvier 1900

17. Juin 1903

Sectio III.

Sectio IV.

9. Janvier 1905

Les auteurs de noms donnés précédemment, mais adoptés par le Sylloge de De Toni seront cités comme tels par analogie avec l'article 42 des règles internationales 1905, p. ex.: "Eunotia arcus Ehrenb. sec. De Toni, Syll. II, p. 790“, ou: "Eun. arcus Ehrenb. sec. D. T." (ou en citant brièvement "Eun. arcus sec. D. T." suffirait peutêtre, quoique cette citation n'est pas tout conforme à l'article 42).

\section{Argumentation concise.}

1. Quoique il est impossible de fonder pour les Cryptogames cellulaires une base unitaire de nomenclature analogue à celle adoptée pour les plantes vasculaires, il est cependant pas opportun de fractionner et diviser cette base plus que nécessaire.

2. Pour les groupes surcités sous no. 1-6, formant comme "algues" un objet unitaire d'études nous possédons dans le Sylloge de De Toni une oeuvre importante de grande exactitude, considérante toute la littérature, embrassante toutes les formes décrites jusqu'au date de publication, élevée au nivean de classifi- 
cation scientifique d'aujourd'hui, bien apte à être employée comme base unitaire de nomenclature.

23 Mars 1909.

de temps.

NB. Une argumentation plus detaillée sera publiée en peu

\section{Neue Cyperaceen.}

Von Ed. Palla (Graz).

V.

(Mit Tafel III.)

Mit Ausnahme von Bulbostylis boliviana entstammen alle hier beschriebenen Arten einer Kollektion ostasiatischer (größtenteils koreanischer) Cyperaceen, die mir Herr H. Leveillé zur Bestimmung übersendet hatte.

\section{Rhynchospora coreana.}

(Fig. 1.)

Halm 6 bis nahezu $7 \mathrm{dm}$ hoch, in der unteren Hälfte etwa $1 / 2 \mathrm{~mm}$ dick, stumpf dreikantig, glatt, im oberen Teile der Infloreszenz $1 / 5-1 / 4 \mathrm{~mm}$ dick, zweikantig, mit einer konvexen und einer konkaven Fläche, an den Kanten sehr schwach rauh. Halmständige Blätter (von den Tragblättern der Seitenspirren abgesehen) 2-3; Scheiden 2 bis nahezu $4 \mathrm{~cm}$ hoch, quer abgestutzt oder sehr schwach konvex vorragend; Blatthäutchen durch eine quer oder schwach konvex verlaufende Linie kaum angedeutet oder fehlend; Spreiten bis $2 \mathrm{dm}$ und darüber lang, $1 \mathrm{~mm}$ oder etwas darüber breit, schmal lineal, flach, glatt, oberwärts dreikantig-rinnig, an den Kanten rauh. Infloreszenz erst im obersten Drittel oder Viertel des Halmes beginnend, aus 2-3 seitenständigen und einer endständigen Spirre zusammengesetzt, 2-4 dm hoch. Spirren weit voneinander entfernt, ohne den Stiel höchstens $1 \mathrm{~cm} \mathrm{~h} \mathrm{o} \mathrm{c} \mathrm{h,} \mathrm{aus} \mathrm{8-3} \mathrm{Ährchen} \mathrm{zusammengesetzt;} \mathrm{die} \mathrm{seitenständigen}$ von ihrem Tragblatt weit überragt, ihr Stiel in der Tragblattscheide eingeschlossen oder kaum über sie hervorra gend. Ährchen (alle in überreifem Zustande vorliegend) $4-5 \mathrm{~mm}$ lang, einzeln, teils kurz gestielt, teils sitzend, mehrblütig. Die großs en Deckblätter breit eiförmig-lanzettlich, kurz stachelspitzig begrannt, rotbraun, $3^{1} / 2-4 \mathrm{~mm} \mathrm{lang}, 1^{3} / 4-2 \mathrm{~mm}$ breit. Frucht (Fig. 1) ohne den Schnabel $1^{1} / 2-1^{3} / 4 \mathrm{~mm}$ lang, 1 oder fast $1 \mathrm{~mm}$ breit, länglich-verkehrteiförmig, bikonvex, schwach querrunzelig, gelb bis braun; Schnabel $1-1^{1 / 2}$ mm lang, an der quer abgestutaten oder schwach konkav ausgeschnittenen Basis 1/2 